\title{
Energy Harvesting of a Magnetostrictive Beam Model Based on Galfenol Alloy
}

\author{
Marek Borowiec ${ }^{1}$, Arkadiusz Syta ${ }^{2}$, Grzegorz Litak ${ }^{3}$ \\ ${ }^{1}$ Department of Applied Mechanics, Lublin University of Technology \\ Lublin, Poland \\ m.borowiec@pollub.pl \\ ${ }^{2}$ Institute of Technological Systems of Information, Lublin University of Technology \\ Lublin, Poland \\ a.syta@pollub.pl \\ ${ }^{3}$ Department of Automation, Lublin University of Technology \\ Nadbystrzycka 36, 20-618 Lublin, Poland \\ g.litak@pollub.pl
}

\begin{abstract}
In the paper the magnetostrictive (MsM) cantilever beam model was analysed. The MsM material was taken into account as Galfenol, the alloy including the Gallium and the Ferrum. The burdened beam system via external excitation produces the magnetic field due to created maximal stress on the external beam fibres. By applied the external coil subsystem, the electrical energy was captured. The main goal of the analysis was to investigate the influence of the beam neutral axis position for energy harvesting efficiency. In case, the neutral axis agrees with the symmetry axis of the magnetostrictive beam, the magnetic fields cancel each other out, while stress is on. This effect disappears while the neutral axis is moved beyond the MsM beam's symmetry. It was estimated the influence of the beam response vibration amplitude on the output electric energy extracted from the system, at increased an effective MsM layer. It was analysed in the vicinity of the first resonance frequency, where the output voltage reached the maximum values.
\end{abstract}

Keywords: magnetostrictive material, energy harvesting, resonance zone

\section{Introduction}

The analysis of the energy harvesting system encompassed the numerical simulations. The output power was calculated while the beam vibrations swept up the first resonance zone by vertical harmonic excitation force (Fig. 1a).

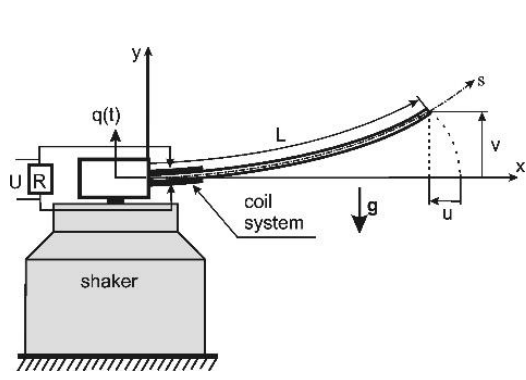

(a)

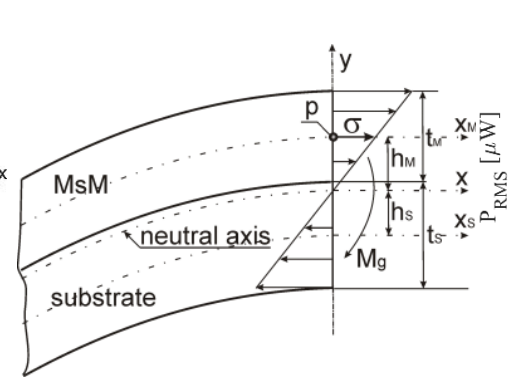

(b)

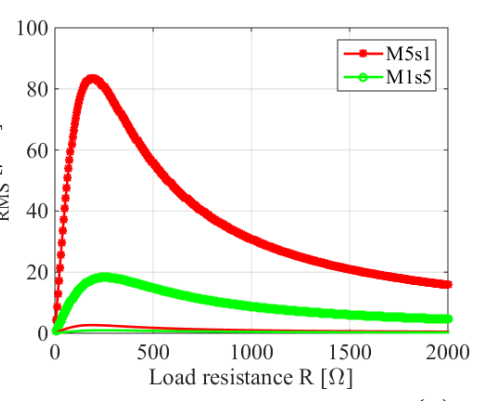

(c)

Fig. 1: The model of the inverted beam with its horizontal and vertical displacements $v$ and $u . L$ is the length of beam, while $x$ is the axis oriented along the beam. $g$ points the gravitational acceleration while, $q$ denotes the kinematic vertical excitation (a), deformation of the sub-beams, magnetostrictive and substrate materials under the bending stress (b) and the influence of the load resistance $R$ on the output power $P$ energy harvesting at the resonance vibration. (c).

The coupled electromechanical model consists of MsM beam with substructure and coil subsystem. The numerical model was derived using the Lagrange's method $[1,2,3]$ and it is given in equations $(1,2)$ : 


$$
\begin{gathered}
{\left[\varrho A N_{1}+\frac{1}{12} \rho A h^{2} N_{4}+\left(\rho A N_{3}+\frac{1}{12} \rho A h^{2} N_{9}\right) v^{2}\right] \frac{d^{2} v}{d t^{2}}+\left(\rho A N_{3}+\frac{1}{12} \rho A h^{2} N_{9}\right) \frac{d v^{2}}{d t} v+\beta \frac{d v}{d t}+E I N_{6}+G i} \\
=-\varrho A N_{2} \frac{d^{2} q}{d t^{2}}
\end{gathered}
$$

where A denotes cross section area of the beam, $\rho$ is materials density, $\mathrm{h}$ is the both beams thickness, $\beta$ is damping coefficient, $E I=E_{M} I_{M}+E_{s} I_{s}$ denotes the Young modulus and area inertia moments of MsM and substructure beams, respectively, $\mathrm{L}$ means the coil inductance and is associated with the pick-up coil around the MsM beam, $R$ is the load resistance, $q(t)$ introduces a vertical excitation to the system, Ni corresponds to the constants (Eq. 5), which depend on the assumed beam shape function and $\mathrm{G}$ is the coupling coefficient (Eq. 3). The values of $G$ depend on the distance between the axis of centroidal MsM beam and the neutral axis $h_{M}$ (Fig. 1b, Eq. 4), the Youngs' modules, thickness of the MsM and substructure beams, the beam shape function $\psi$, the coil numbers $\mathrm{N}$, and the magneto - mechanical coefficient $\boldsymbol{d}$.

$$
\begin{gathered}
G=\frac{E_{M} A_{M} h_{M} N \boldsymbol{d}}{l} \int_{0}^{l} \psi^{\prime \prime}(s) d s \\
h_{M}=\frac{t_{s}\left(t_{M}+t_{s}\right)}{2\left(\frac{E_{M}}{E_{S}} t_{M}+t_{s}\right)}
\end{gathered}
$$

The system parameters applied in analysis are listed in Tab 1.

Table 1: The system parameters.

\begin{tabular}{|l|l|}
\hline \multicolumn{1}{|c|}{ Symbol and value } & \multicolumn{1}{c|}{ Description } \\
\hline$l=0.185[\mathrm{~m}]$ & the beams length \\
\hline$A=b \times h=72\left[\mathrm{~mm}^{2}\right]$ & the cross section of the beams \\
\hline$\rho=7864\left[\mathrm{~kg} / \mathrm{m}^{3}\right]$ & density of the MsM and substructure beam masses \\
\hline$E_{M}=40[\mathrm{GPa}]$ & Young modulus of MsM material \\
\hline$E_{S}=191[\mathrm{GPa}]$ & Young modulus of substructure \\
\hline$R=(5-2000)[\Omega]$ & the load resistance range \\
\hline$L=352[\mathrm{mH}]$ & the pick-up coil inductance \\
\hline$N=3000$ & the coil number \\
\hline$h_{M}=(1-5)[\mathrm{mm}]$ & the MsM thickness \\
\hline$A_{M}=b \times h_{M}=(12-60)\left[\mathrm{mm}^{2}\right]$ & the MsM cross section \\
\hline $\boldsymbol{d}=1.5 \times 10^{-9}[\mathrm{~m} / \mathrm{A}]$ & the magneto - mechanical coefficient \\
\hline$G=(0.5-1.5)[\mathrm{N} / \mathrm{A}]$ & the coupling parameter (gyrator) range \\
\hline
\end{tabular}

Corresponding constants depend on shape function of the beam are given by

$$
\begin{array}{lr}
N_{1}=\int_{0}^{l} \psi(s)^{2} d s=1.0 l[\mathrm{~m}], & N_{2}=\int_{0}^{l} \psi(s) d s=0.78 l[\mathrm{~m}], \\
N_{3}=\int_{0}^{l}\left(\int_{0}^{s}\left(\psi^{\prime}(s)^{2} d s\right)^{2} d s=4.60 / l\left[\mathrm{~m}^{-1}\right]\right. & N_{4}=\int_{0}^{l} \psi^{\prime}(s)^{2} d s=4.65 / l\left[\mathrm{~m}^{-1}\right], \\
N_{6}=\int_{0}^{l} \psi^{\prime \prime}(s)^{2} d s=12.36 l^{3}\left[\mathrm{~m}^{-3}\right] & N_{9}=\int_{0}^{l} \psi^{\prime}(s)^{4} d s=28.79 l^{3}\left[\mathrm{~m}^{-3}\right]
\end{array}
$$


In Fig. 1c is shown, that the energy harvesting efficiency depends significantly on the loader conditions. It is important to estimate an optimal loader resistance, whereby the system charged the maximal output power $P$ [4]. For chosen the optimal resistance $R_{\text {opt }}$, the numerical analysis have been conducted.

\section{Numerical Results}

The analysis of an energy harvesting efficiency has been investigated with respect to different proportion of the MsM material and substructure of the system. In Figures $2 \mathrm{a}-\mathrm{d}$ is shown the output behaviour of the system for swept up of the excitation frequency at eight different excitation amplitude levels. First four amplitudes are assumed relatively small (Figs. 2a,b). In case the beams proportion thickness MsM is $1 \mathrm{~mm}$ and substructure is $5 \mathrm{~mm}$ (Figs. 2a), the output power threshold regarding to excitation amplitude is $6.33 \%, 25.32 \%, 56.77 \%, 100 \%$ and in inversely beams proportion thickness case (Figs. 2b), this threshold is $6.26 \%, 25.06 \%, 56.35 \%, 100 \%$, respectively.

Regarding to excitation amplitude range of the beam around $y_{e}=(0.25-1) \mathrm{mm}$, the output power behaviour is rather linear although the system equation (1) consists nonlinear terms. This can be explained by stiffness of the beam layers. But in case the excitation amplitude is raised up to $y_{e}=10 \mathrm{~mm}$ the system behaviour demonstrated a no-symmetry in the power frequency response, simultaneously increasing the energy harvesting. While $y_{e}$ increased ten times, the maximal output power has grown about forty times from $P=0.98 \mu W$ to $P=38.33 \mu W$ in analysed the first beams proportion (see Figs. 2a and 2c), and corresponding about sixty times from $P=2.68 \mu \mathrm{W}$ to $P=165.43 \mu \mathrm{W}$ in the second beam proportion (see Figs. $2 \mathrm{~b}$ and $2 \mathrm{~d})$. Moreover at higher range of the excitation amplitudes $y_{e}=(4-10) \mathrm{mm}$, the system reveals that resonance point of the excitation frequency moves left in both cases of the beams thickness proportion.

Comparing levels of gained power, the $P_{R M S}$ values are significantly higher in the second cases shown in Fig. 2b and $2 \mathrm{~d}$. Such response of the system suggests that increasing thickness of the MsM layer regarding to the substructure causes increasing the energy harvested by the system.

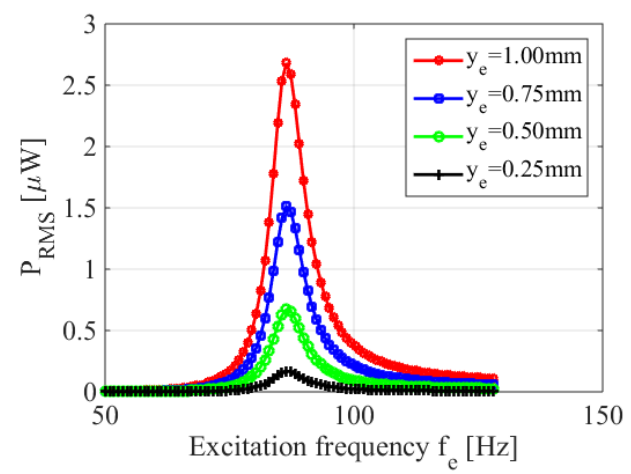

(a)

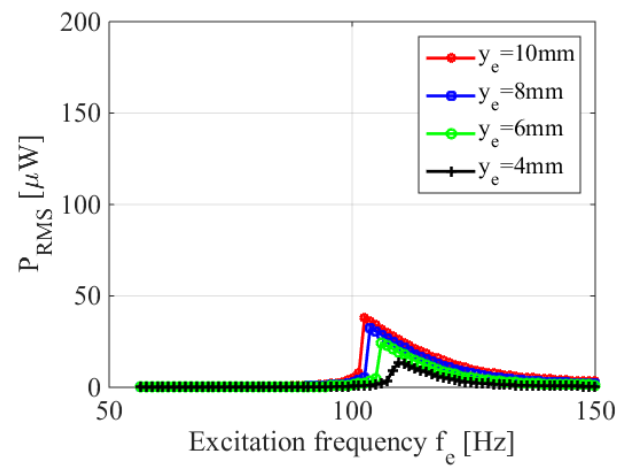

(c)

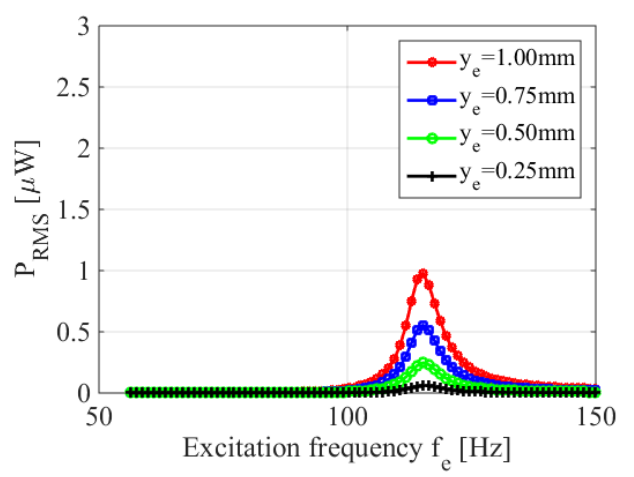

(b)

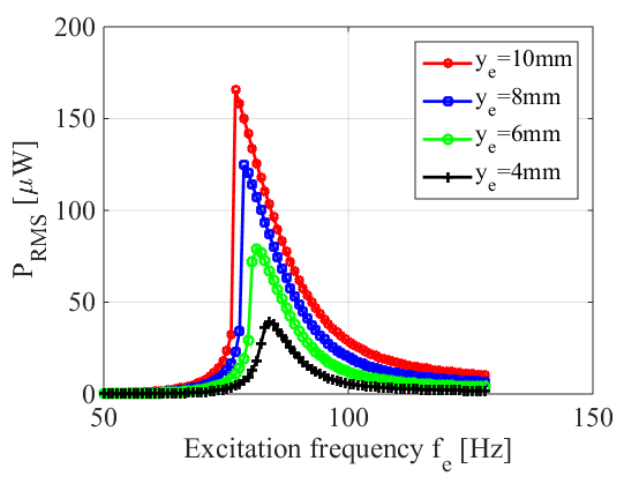

(d)

Fig. 2: The output root mean square of harvested power at optimal Ropt load resistances in vicinity of the first resonance zone. The thickness of MsM and substructure are $1 \mathrm{~mm}$ and $5 \mathrm{~mm}$ case (a) and inversely $5 \mathrm{~mm}$ and $1 \mathrm{~mm}$, case (b). 


\section{Conclusion}

The influence of bending moments on stress appeared in magnetostrictive beam revealed the different energy harvesting efficiency while the neutral axis is moved away from the MsM beam symmetry axis. Applying combination of magnetostrictive and substructure beams thickness proportions, it is reported the root mean square of an output power changes significantly. Moreover, it is observed a slight movement of resonance point while excitation amplitude is adequately increased. Presented results could be useful in vibration shells of any systems. That solution of energy harvesting could be applied as an additional sheathing in special manufactured shells of any mechanical devices. While the excitation conditions occurring, the effective shell will convert the mechanical energy into an electrical one. It could charge small sensors, eliminating wires of an external power support.

\section{Acknowledgements}

The work has been supported by the National Science Centre under the grant Agreement No. DEC2013/11/D/ST8/03308.

\section{References}

[1] M. I. Friswell, A. S. Faruque, O. Bilgen, S. Adhikari, A. Lees and G. Litak: "Non-linear piezoelectric vibration energy harvesting from a vertical cantilever beam with tip mass," Journal of Intelligent Material Systems and Structures. vol. 23, no. 13, pp. 1505-1512, 2012.

[2] M. Borowiec, G. Litak, M. I. Friswell, A. S. Faruque, S. Adhikari, A. Lees and O. Bilgen: "Energy harvesting in piezoelastic systems driven by random excitation," International Journal of Structural Stability and Dynamics., vol. 13 no. 7, pp. 1340006:1-1340006:11, 2013.

[3] M. Borowiec: "Energy harvesting of cantilever beam system with linear and nonlinear piezoelectric model," The European Physical Journal ST, vol. 224, pp. 2771-2785, 2015.

[4] L. Wang and F.G. Yuan: "Vibration energy harvesting by magnetostrictive material," Smart Mater. Struct. vol. 17, 045009, pp.14, 2008. 